\title{
SCIDiC
}

\author{
International Journal of Dentistry and Oral Science (IJDOS) \\ ISSN: 2377-8075
}

\section{To Evaluate the Efficacy of New Brush Covered Irrigation Needle in Removing Root Canal Debris In Vitro: A Scanning Electron Microscopy Study}

Research Article

Piyush Oswal ${ }^{1}$, Santosh S. Martande ${ }^{2 *}$, Suresh Shenvi ${ }^{3}$

Department of Conservative Dentistry and Endodontics, Dr. D Y Patil Vidyapeeth, Pimpri, Pune, 400 018, Maharashtra, India.

${ }^{2}$ Department of Periodontology, Dr. D Y Patil Vidyapeeth, Pimpri, Pune, 400 018, Maharashtra, India.

${ }_{3}^{3}$ Department of Conservative Dentistry and Endodontics, KLE VK Institute of Dental Sciences, Belgaum, KAHER, Karnataka, India.

\section{Abstract}

Objective: The main purpose of the study was to compare the efficacy of NaviTip and NaviTip FX in debriding the walls of the canals using a Scanning Electron Microscope (SEM). The secondary purpose of the study was to evaluate if use of $17 \%$ EDTA along with $5.25 \% \mathrm{NaOCl}$ had an additional effect in root canal debridement.

Material and Methods: Access cavity preparations were performed in 40 extracted incisor teeth. All the teeth were randomly allotted to any of 4 groups consisting of 10 teeth- Group 1: Irrigation with $1 \mathrm{ml}$ of $5.25 \% \mathrm{NaOCl}$ with NaviTip needle after each instrumentation; Group 2: Irrigation with $1 \mathrm{ml}$ of $5.25 \% \mathrm{NaOCl}$ using NaviTip FX with a manual left and right rotary motion combined with up and down motions, and a brushing action on dentin walls after each instrumentation; Group 3: Irrigation with $1 \mathrm{ml}$ of $5.25 \% \mathrm{NaOCl}$ followed by $1 \mathrm{ml}$ of $17 \%$ EDTA with NaviTip needle after each instrumentation; Group 4: Irrigation with $1 \mathrm{ml}$ of $5.25 \% \mathrm{NaOCl}$ followed by $1 \mathrm{ml}$ of $17 \%$ EDTA using NaviTip FX with a manual left and right rotary motion combined with up and down motion, and a brushing action on dentin walls after each instrumentation. Later each selected sample was further split horizontally into three halves as coronal (10 $\mathrm{mm}$ from apex), middle ( $5 \mathrm{~mm}$ from apex) and apical (1 $\mathrm{mm}$ from apex) thirds and treated with sputter-coated withgold using fine-coat ion sputter JFC-1100. Samples were analyzedfinally by SEM analysis.

Result: All the groups showed significantly lesser smear layer at $10 \mathrm{~mm}$ when compared to $5 \mathrm{~mm}$ and $1 \mathrm{~mm}$. Further, it signifies that NaviTip FX (Group 4) when used along with 5.25\% NaOCL and 17\% EDTA removed more debris as compared to Group 1 and 2 at $10 \mathrm{mmand} 5 \mathrm{~mm}$; and removed more debris at $1 \mathrm{~mm}$ compared to Group 3.

Conclusion: Navi Tip could be replaced by Navi Tip FX for the purpose of cleaning and removal of smear layer. EDTA, which is considered as one of the best irrigants for removal dentinal debris may be ineffective in some narrower canals and could be adjuncted with manual irrigation devices such as Navi Tip FX to improve its action.

Keywords: Root Canal Debridement; Root Canal Irrigation; 5.25\% NaOCl; 17\% EDTA; Scanning Electron Microscopy.

\section{Introduction}

The core of endodontic instrumentation lies in the cleaning, shaping and canal preparation. It is contemplated as an important step as it removes the vital and necrotic pulp which contains the microflora that can cause failure of the endodontic treatment $[1,2]$. Furthermore, the debris that contains the chips of dentin, other organic and inorganic content block the canals for proper flow of sealer leading to a poor obturation. Several studies have been conducted and regardthat the debris should be completely removed for a successful endodontic treatment. For this purpose, the canals are thoroughly instrumented with both hand and rotary instruments in an effort to remove the debris totally $[3,4]$.

Literature has multiple evidences which state that mechanical instrumentation alone cannot remove the debris totally, hence, chemical methods have also been advocated for successful removal of debris and disinfection of the canals [5]. Even if the debris has been successfully removed, the instrumented canal walls leave a 1 to $2 \mu$ thin layer called as smear layer which clogs the dentinal tubules, thus preventing the flow of sealer [6]. The use of rotary instruments also leaves certain areas such as isthmuses, cul-de-sacs and canal fins inaccessible 7.1 to $6 \% \mathrm{NaOCl}$ and $17 \%$ EDTA are considered to be the best intracanal irrigants that are used as an adjunct to mechanical debridement to remove

*Corresponding Author:

Dr. Santosh S. Martande MDS,

Department of Periodontology, Dr. D Y Patil Vidyapeeth, Pimpri, Pune, 400 018, Maharashtra, India

Tel: 9890353072

E-mail: santoshmartande@gmail.com

Received: November 18, 2020

Accepted: December 15, 2020

Published: December 16, 2020

Citation: Piyush Oswal, Santosh S. Martande, Suresh Shenvi. To Evaluate the Efficacy of New Brush Covered Irrigation Needle in Removing Root Canal Debris In Vitro: A Scanning Electron Microscopy Study Int J Dentistry Oral Sci. 2020;7(12):1211-1215. doi: http://dx.doi.org/10.19070/2377-8075-20000239

Copyright: Santosh S. Martande ${ }^{\circ} 2020$. This is an open-access article distributed under the terms of the Creative Commons Attribution License, which permits unrestricted use, distribution and reproduction in any medium, provided the original author and source are credited. 
the smear layer. Most importantly, the irrigant should come into contact with the walls of the canals for which irrigation devices are used. The manual irrigation devices are cost effective and aid in irrigating the canals as effectively as powered irrigation devices.

NaviTip and NaviTip FX are the two manual irrigation devices that are used to deliver intracanal irrigants and act upon canal walls to remove the smear layer. The NaviTip is available in various lengths varying from 17 to $27 \mathrm{~mm}$ in 29 and 30 gauges. It consists of delivery cannula which is rigid from base to the middle third beyond which is flexible in the apical third. This design facilitates for easy movement of the tip through curved canals. The latter variant i.e. NaviTip FX consists of same design but is flocked with an irregularly grit surface that helps for mechanical debridement while irrigating the walls of the canals.

Several studies have been performed using NaviTip and NaviTip FX individually and have proven to be efficacious. These devices have gained importance as they deliver intracanal irrigant whilst mechanically removing the smear layer or debris. According to our knowledge, very meagre evidence is available comparing the efficacy of these two devices. So, the main purpose of the study was to compare the efficacy of NaviTip and NaviTip FX in debriding the walls of the canals using a Scanning Electron Microscope (SEM). The secondary purpose of the study was to evaluate if use of $17 \%$ EDTA along with $5.25 \% \mathrm{NaOClhad}$ an additional effect in root canal debridement.

\section{Material and Methods}

The study was performed as an invitro study using scanning electron microscopy. Forty anterior incisors were collected from Department of Oral and Maxillofacial Surgery and were stored in the running water until the teeth were subjected to microscopy. Healthy, non-carious, incisors which did not show any signs of pathologic root resorption, fractures and double roots in mesiodistal radiographs were chosen into the study.

Access cavity preparations were performed to the pulpchambers and a \#15 K-type file was inserted into the canaluntil the tip was just visible at the apical foramen. The length of the file was measuredand $1 \mathrm{~mm}$ was subtracted from this length to establish working length after which sticky wax was used to seal the tooth apex. The initial canal enlargement was done using Gates Glidden drills size 2, 3 and 4. Instrumentation was performed using crown down technique with k-files size \#15, 20, 25, 30, 35 and 40 to theworking length. In between the instrumentation the canals were irrigated using one of the following protocols explained below.

All the teeth were divided and randomly allotted to any of the following 4 groups each consisting of 10 teeth- Group 1: Irrigation was done with $1 \mathrm{ml}$ of $5.25 \% \mathrm{NaOCl}$ with NaviTip needle aftereach instrumentation; Group 2: Irrigation was done with $1 \mathrm{ml}$ of $5.25 \% \mathrm{NaOCl}$ using NaviTip FX with amanual left and right rotary motion combined with up and down motions, and a brushing action on dentin walls after each instrumentation; Group 3: Irrigation was done with $1 \mathrm{ml}$ of $5.25 \% \mathrm{NaOCl}$ followed by $1 \mathrm{ml}$ of $17 \%$ EDTA with NaviTip needle after each instrumentation; Group 4: Irrigation was done with $1 \mathrm{ml}$ of $5.25 \% \mathrm{NaOCl}$ followed by $1 \mathrm{ml}$ of $17 \%$ EDTA using NaviTip FX with a manual left and right rotary motioncombined with up and down motion, and a brushing action on dentin wallsafter each instrumentation. After canal preparation all the canals were dried with paper points and vertical grooves were placed bucco-lingually using carborundum discs. The teeth were separated using chisel-malletand that half of the tooth with a more visible apex was used. Each selected sample was further split horizontally into three halves as coronal $(10 \mathrm{~mm}$ from apex), middle ( $5 \mathrm{~mm}$ from apex) and apical ( $1 \mathrm{~mm}$ from apex) thirds with markings using a sharp instrument. The samples were treated with sputter-coated withgold using fine-coat ion sputter JFC-1100 (Fine coat ion sputter JFC-1100, JEOL Ltd., Tokyo, Japan), and then evaluated using the SEM (JeolJSM-6360 LV, JEOL Ltd.). and Paque's protocol was used for standardisation of the microscopy. Debris on the canal wall was evaluated using the following scoring system:

Score 1: Clean root canal, only few small debris particles.

Score 2: Few small isles of debris covering less than $25 \%$ of the roocanal wall.

Score 3: Many accumulations of debris covering more than $25 \%$ buless than $50 \%$ of the root canal wall.

Score 4: More than $50 \%$ of the root canal wall covered by debris.

\section{Statistical Analysis}

The results were statisticallyanalysed using theKruskal Walls and Mann-Whitney U-test at significance levelp $<0.05$ using IBM SPSS software version 21.

\section{Results}

The inter group comparisons were done using the Kruskal Walls test while pairwise comparisons were done using Mann-Whitney U test. Figures 1, 2, 3 and 4 depict the Scanning Electron Microscopy images of the canal walls for the amount of smear layer removed. All the groups showed significantly lesser smear layer at $10 \mathrm{~mm}$ when compared to $5 \mathrm{~mm}$ and $1 \mathrm{~mm}$. Further, it signifies that NaviTip FX (Group 4) when used along with 5.25\% NaOCLand 17\% EDTA removed more debris as compared to Group 1 and 2 at $10 \mathrm{mmand} 5 \mathrm{~mm}$; and removed more debris at $1 \mathrm{~mm}$ compared to Group 3.

The pair wise inter group comparisons of Mann Whitney test have showed that at $10 \mathrm{~mm}$ away from apex, Groups 2, 3 and 4 were cleaner than Group 1; Groups3 and 4 were cleaner that Group 2 and showed statistical significance while there was no difference between Group 3 and 4 . At $5 \mathrm{~mm}$ away from root apex, Groups 3 and 4 were cleaner than Group 1 while only Group 4 was cleaner than Group 2 and showed statistical significance. The remaining pairwise comparisons remained statistically insignificant. At $1 \mathrm{~mm}$ away from apex, only Group 4 had significantly cleaner canals than all other groups while the remaining pairwise comparisons remained insignificant.

\section{Discussion}

This in vitro study using a scanning electron microscope was performed primarily to evaluate the difference in the working efficiency between NaviTip and NaviTip FX to remove the smear layer from the canal walls of the root. 40 teeth without any pathologic changes have been selected for the study. All the teeth were 
Table 1. Intra group comparisons at $10 \mathrm{~mm}, 5 \mathrm{~mm}$ and $1 \mathrm{~mm}$ from apex using Kruskal Walls test.

\begin{tabular}{|c|c|c|c|c|}
\hline & $10 \mathrm{~mm}$ & $5 \mathrm{~mm}$ & $1 \mathrm{~mm}$ & Kruskal Walls p value \\
\hline Groups & Mean \pm SD & Mean \pm SD & Mean \pm SD & \multirow{5}{*}{$\begin{array}{c}\mathrm{P}<0.001 \text {, HS (Highly Signifi- } \\
\text { cant) }\end{array}$} \\
\hline 1 & $3.7 \pm 0.48$ & $3.3 \pm 0.68$ & $3.3 \pm 0.48$ & \\
\hline 2 & $2.9 \pm 0.32$ & $2.8 \pm 0.42$ & $3.4 \pm 0.52$ & \\
\hline 3 & $2.1 \pm 0.57$ & $2.6 \pm 0.7$ & $2.9 \pm 0.74$ & \\
\hline 4 & $1.7 \pm 0.68$ & $2.2 \pm 0.42$ & $1.8 \pm 0.79$ & \\
\hline
\end{tabular}

Table 2. Pair wise comparisons using Mann Whitney test at $10 \mathrm{~mm}, 5 \mathrm{~mm}$ and $1 \mathrm{~mm}$ away from apex.

\begin{tabular}{|c|c|c|c|c|c|c|c|c|c|c|c|c|c|}
\hline & \multicolumn{4}{|c|}{ At $\mathbf{1 0} \mathbf{~ m m}$} & \multicolumn{4}{c|}{ At $\mathbf{5} \mathbf{~ m m}$} & \multicolumn{4}{c|}{ At $\mathbf{~ m m}$} \\
\hline Groups & $\mathbf{1}$ & $\mathbf{2}$ & $\mathbf{3}$ & $\mathbf{4}$ & $\mathbf{1}$ & $\mathbf{2}$ & $\mathbf{3}$ & $\mathbf{4}$ & $\mathbf{1}$ & $\mathbf{2}$ & $\mathbf{3}$ & $\mathbf{4}$ \\
\hline 1 & - & $0.001 * *$ & $0.001 * *$ & $0.001 * *$ & - & 0.06 & $0.04 *$ & $0.001 * *$ & - & 0.65 & 0.18 & $0.001^{* *}$ \\
\hline 2 & - & - & $0.002 * *$ & $0.001 * *$ & - & - & 0.34 & $0.009 * *$ & - & - & 0.11 & $0.001 * *$ \\
\hline 3 & - & - & - & 0.15 & - & - & - & 0.15 & - & - & - & $0.001 * *$ \\
\hline 4 & - & - & - & - & - & - & - & - & - & - & - & - \\
\hline
\end{tabular}

** indicates highly significant

* indicates significant

Figure 1.

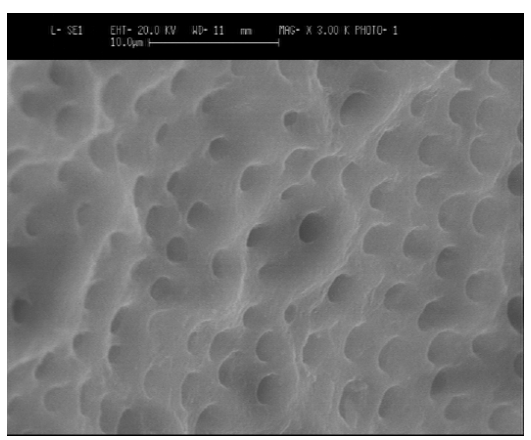

Figure 2.

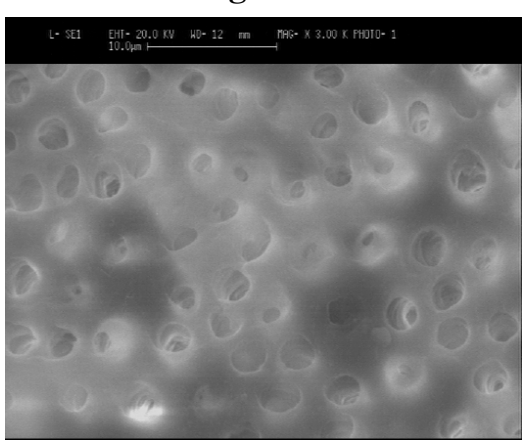

Figure 3.

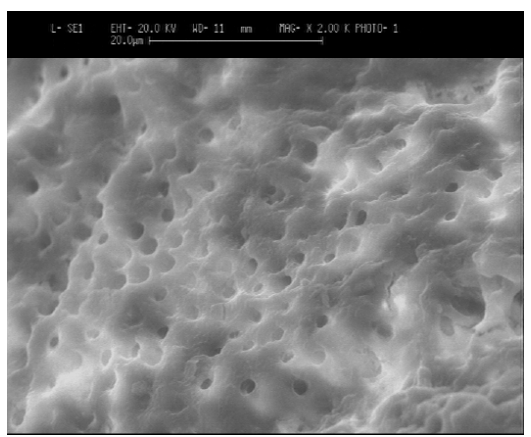


Figure 4 .

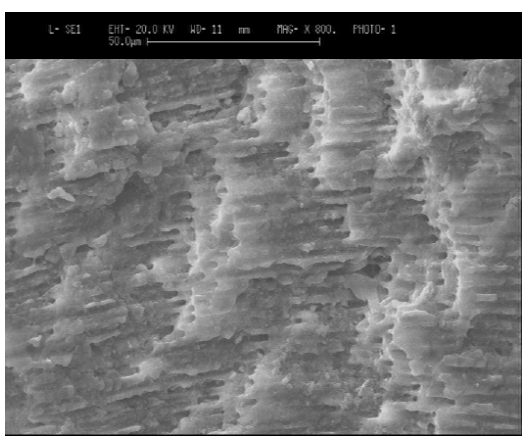

Flowchart 1. Classification of Irrigation Devices.

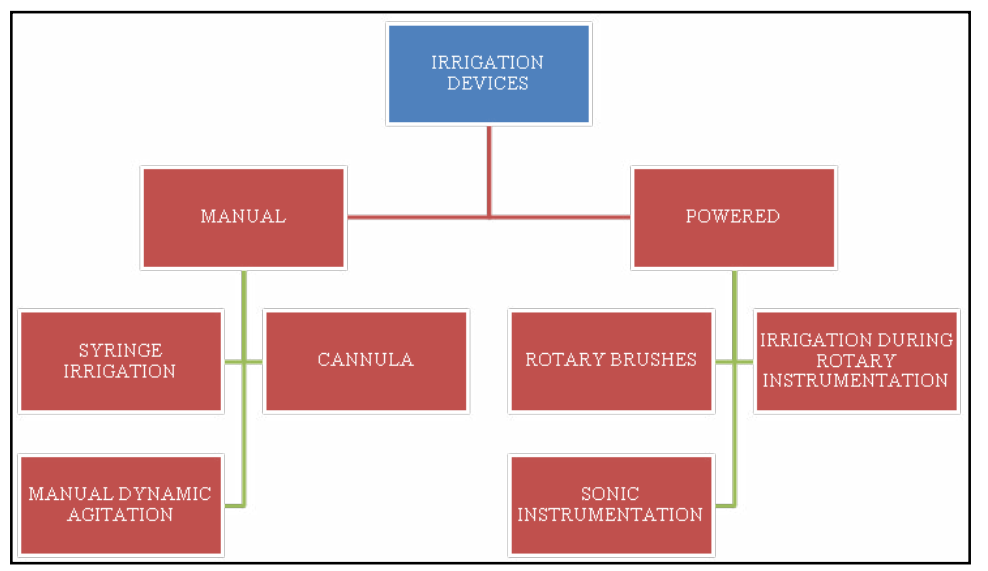

endodontically treated using the crown down technique. To simulate the clinical condition, the apices of the teeth were sealed using sticky wax. Later, they were divided into three sections as coronal third (10mm from apex), middle third (5mm from apex) and apical third ( $1 \mathrm{~mm}$ from apex). The examiners were blinded regarding the nature of study during the microscopic examination to standardise the procedure and to avoid examination bias.

Results of the study have shown that all the four groups removed the smear layer better in the coronal third when compared to middle third and apical third but there were differences in the amount of smear layer removed when compared with the other groups. Group 4 that was instrumented with NaviTip FX and treated with $17 \%$ EDTA showed superior results than the other four groups at all the three levels. This provides insight into the better capabilities of the NaviTipFX in removing the smear layer. Solaiman et al have conducted a scanning electron microscopic study on thirty single rooted teeth using Navi Tip FX and 5\% $\mathrm{NaOCl}$ against a control group which did not have bristles on the irrigation device [8]. NaviTip FX showed superior results in terms of removal of smear layer in the coronal third of the canals8. Similarly, several studies have been performed individually using NaviTip and NaviTip FX and both the instruments have performed well and were able to significantly remove the smear layer. In this present study, Navi Tip FX has performed superiorly than Navi Tip that is evident from the results i.e. table 2. The apical third of the root showed canals walls with less smear layer than the other three groups. Although 17\% EDTA helps in lubricating the canal walls and helps in easy smear layer removal that is evident from the pairwise comparison between Group 3 and 4 at $10 \mathrm{~mm}$ and $5 \mathrm{~mm}$ away from apex, its action could have been be nullified at the apical third of the root where the NaviTip FX performed well even if $17 \%$ EDTA was not applied. This is evident from the pairwise comparisons between Group 4 and other three groups at the api- cal third (1mm away from the apex).

The superiority of NaviTip FX over NaviTip lies in the design and the method in which the instrument is used. NaviTip FX has an irregularly grit surface that helps in removal of smear layer and prevents clogging of the apical third with dentinal debris. The up and down motion combined with brush like strokes prevents from creating any ledges that might lead to failure of the endodontic treatment. Ethylene Diamine Tetra Acetic Acid (EDTA) is a chelating agent that forms disodium chelate salts with calcium. This property helps in removal of smear layer and several studies have proven its efficacy [9-11]. The probable reason for EDTA not performing equivalent to the test group is because of the narrower canals that might have caused clogging in the apical third of the root and this could be overcome using the Navi Tip FX.

According to our knowledge, very few studies have been conducted comparing the Navi Tip and Navi Tip FX and this study could shed some light on the superiority of the Navi Tip FX. The limitations of this study could be considered as the future prospective where multirooted, curved and longer canals should be irrigated with this new device to test its effectiveness for regular clinical use.

\section{Conclusion}

Within the limitations of present study, it can be concluded that the Navi Tip could be replaced by Navi Tip FX for the purpose of cleaning and removal of smear layer. EDTA, which is considered as one of the best irrigants for removal dentinal debris may be ineffective in some narrower canals and could be adjuncted with manual irrigation devices such as Navi Tip FX to improve its action. 


\section{References}

[1]. Wong R. Conventional endodontic failure and retreatment. Dent. Clin. N. Am. 2004 Jan;48(1):265-89.

[2]. Basmadjian-Charles CL, Farge P, Bourgeois DM, Lebrun T. Factors influencing the long-term results of endodontic treatment: a review of the literature. Int Dent J. 2002 Apr;52(2):81-6.Pubmed PMID: 12013255.

[3]. Khedmat S, Shokouhinejad N. Comparison of the efficacy of three chelating agents in smear layer removal. J Endod. 2008 May;34(5):599-602.Pubmed PMID: 18436043

[4]. Kuah HG, Lui JN, Tseng PS, Chen NN. The effect of EDTA with and without ultrasonics on removal of the smear layer. J Endod. 2009 Mar $1 ; 35(3): 393-6$

[5]. Ferreira RB, Alfredo E, Porto de Arruda M, Silva Sousa YT, Sousa-Neto MD. Histological analysis of the cleaning capacity of nickel-titanium rotary instrumentation with ultrasonic irrigation in root canals. Aust Endod J. 2004 Aug;30(2):56-8.Pubmed PMID: 15378973.

[6]. Garip Y, Sazak H, Gunday M, Hatipoglu S. Evaluation of smear layer re- moval after use of a canal brush: an SEM study. Oral Surg Oral Med Oral Pathol Oral Radiol Endod. 2010 Aug 1;110(2):e62-6.

[7]. Walton RE. Histologic evaluation of different methods of enlarging the pulp canal space. J Endod. 1976 Oct;2(10):304-11.Pubmed PMID: 1068207.

[8]. Al-Hadlaq SM, Al-Turaiki SA, Al-Sulami U, Saad AY. Efficacy of a new brush-covered irrigation needle in removing root canal debris: a scanning electron microscopic study. J Endod. 2006 Dec;32(12):1181-4.Pubmed PMID: 17174678

[9]. Wu L, Mu Y, Deng X, Zhang S, Zhou D. Comparison of the effect of four decalcifying agents combined with $60^{\circ} \mathrm{C} 3 \%$ sodium hypochlorite on smear layer removal. J Endod. 2012 Mar;38(3):381-4.Pubmed PMID: 22341079.

[10]. Prado M, Gusman H, Gomes BP, Simão RA. Scanning electron microscopic investigation of the effectiveness of phosphoric acid in smear layer removal when compared with EDTA and citric acid. J Endod. 2011 Feb;37(2):2558.Pubmed PMID: 21238813.

[11]. Dai L, Khechen K, Khan S, Gillen B, Loushine BA, Wimmer CE, Gutmann JL, Pashley D, Tay FR. The effect of QMix, an experimental antibacterial root canal irrigant, on removal of canal wall smear layer and debris. J Endod. 2011 Jan 1;37(1):80-4 